This item was submitted to Loughborough's Research Repository by the author.

Items in Figshare are protected by copyright, with all rights reserved, unless otherwise indicated.

\title{
Traveling waves and pulses in a one-dimensional network of excitable
} integrate-and-fire neurons

PLEASE CITE THE PUBLISHED VERSION

LICENCE

CC BY-NC-ND 4.0

\section{REPOSITORY RECORD}

Bressloff, P.C.. 2019. "Traveling Waves and Pulses in a One-dimensional Network of Excitable Integrate-andfire Neurons". figshare. https://hdl.handle.net/2134/826. 


\title{
Traveling waves and pulses in a one-dimensional network of excitable integrate-and-fire neurons*
}

\author{
Paul C. Bressloff \\ Nonlinear and Complex Systems Group \\ Department of Mathematical Sciences, Loughborough University \\ Loughborough, Leicestershire LE11 3TU, U. K.
}

April 19, 1999

\begin{abstract}
We study the existence and stability of traveling waves and pulses in a one-dimensional network of integrate-and-fire neurons with synaptic coupling. This provides a simple model of excitable neural tissue. We first derive a self-consistency condition for the existence of traveling waves, which generates a dispersion relation between velocity and wavelength. We use this to investigate how wave-propagation depends on various parameters that characterize neuronal interactions such as synaptic and axonal delays, and the passive membrane properties of dendritic cables. We also establish that excitable networks support the propagation of solitary pulses in the long-wavelength limit. We then derive a general condition for the (local) asymptotic stability of traveling waves in terms of the characteristic equation of the linearized firing time map, which takes the form of an integro-difference equation of infinite order. We use this to analyze the stability of solitary pulses in the long-wavelength limit. Solitary wave solutions are shown to come in pairs with the faster (slower) solution stable (unstable) in the case of zero axonal delays; for non-zero delays and fast synapses the stable wave can itself destabilize via a Hopf bifurcation.
\end{abstract}

\section{Introduction}

A number of recent experimental studies have revealed the propagation of traveling bursts of activity in slices of excitable neural tissue taken from the cortex (Chervin et al 1988; Golomb and Amitai 1997), hippocampus (Traub et al 1993) and thalamus (Kim et al 1995; Golomb et al 1996; Destexhe et al 1996). The underlying mechanism for propagation of these waves is thought to be synaptic in origin rather than diffusive as in the propagation of action potentials. Computational models of synaptically generated waves have also been developed, in which neural tissue is treated

${ }^{*}$ J. Math. Biol. (submitted) 
as a one-dimensional continuum (Traub et al 1993; Golomb et al 1996; Destexhe et al 1996: Golomb and Amitai 1997). These various studies indicate that wave propagation in cortical (and hippocampal) slices only occurs if the synaptic strength of neuronal interactions exceeds some threshold; well beyond threshold the velocity is approximately linear in the coupling (see eg. Golomb and Amitai 1997). Moreover, multiple forms of discharge patterns are observed to propagate at the same parameter values. Selection of a particular pattern occurs through the initial conditions such as the strength of the initial stimulus. Interestingly, there is not much variation in the speed as a function of the number of spikes that propagate. The mechanism of wave propagation in thalamic slices appears to be different from cortical and hippocampal tissue; thalamic waves propagate in a lurching manner resulting in an approximately logarithmic dependence of velocity on synaptic coupling strength and there is no minimal wave velocity (Golomb et al 1996).

An important question concerns how the velocity of a wave depends on the synaptic and intrinsic cellular properties of the neurons. This is difficult to extract from detailed computational models. However, Ermentrout (1998) has established that a simple integrate-and-fire (IF) model of a neuron captures much of the process underlying an excitation wave in cortical slices. This is due to the fact that the velocity of a wave in the case of strong synaptic coupling is essentially independent of the ionic details of cell membranes, depending mainly on the integration rise time from the resting potential to threshold. Ermentrout (1998) has used the analytically tractable IF model to derive a number of scaling laws for the velocity of a single activity pulse as a function of various parameters of the system. In particular, he has shown that velocity scales linearly with the range of synaptic interactions and the synaptic decay rate, but exhibits a non-trivial power-law with respect to the synaptic coupling strength. He has also established numerically that similar scaling laws hold for the more detailed computational models.

In this paper we further develop the analysis of IF models of cortical wave propagation. In particular, we show how the existence and stability of solitary pulses may be studied by taking the long-wavelength limit of periodic traveling wave solutions. This is important for a number of reasons. First, it enables us to prove that the solitary wave solutions analyzed by Ermentrout (1998) are stable. Second, it establishes that the velocity of a wave is approximately independent of wavelength (at least for long wavelength solutions). This is at least suggestive of the approximate velocity-independence of the various discharge patterns observed in more detailed computational models (Golomb and Amitai 1997). As highlighted by Ermentrout (1998), a major unsolved problem is the detailed nature of the effects of re-excitation on wave propagation. Our analysis provides some insight into the transition between single and repetitive firing states. A third aspect of our analysis is to confirm the existence of scaling laws for the velocity as a function of synaptic strength for more general forms of synaptic interaction that includes the effects of passive dendrites.

We begin in section 2 by introducing a continuum model of cortical tissue based on a onedimensional network of IF neurons with synaptic coupling. An IF neuron fires a spike whenever 
its membrane potential reaches some threshold, and immediately after firing the membrane potential is reset to some resting level (Keener et al 1981). Each neuron is assumed to receive a constant external bias $I_{0}$ such that, in the absence of any synaptic coupling, the neuron oscillates (periodically fires and resets) when $I_{0}>h$ (oscillatory regime) and is quiescent (in the absence of additional stimulation) when $I_{0}<h$ (excitable regime). Here $h$ denotes the threshold for firing. We define a (periodic) traveling wave as a phase-locked state in which the sequence of firing times of the neuron at $x \in \mathbb{R}$ satisfies $T_{n}(x)=(n+k x) T$ for integer $n$, where $k$ is the wavenumber and $c=1 / k T$ is the velocity of the wave. In other words, all the neurons fire at regular intervals of length $T$ but the firing times are shifted according to the phase $\theta(x)=k x$. A self-consistency condition for the existence of a traveling wave is derived by considering the nonlinear mapping of the neuronal firing times along the lines of Bressloff and Coombes (1998a$\mathrm{c}, 1999)$. This yields a dispersion relation for the velocity $c=c(k)$. We calculate the resulting dispersion curves as a function of various parameters characterizing neuronal interactions. These include the rise-time of synaptic response, discrete axonal propagation delays, and the passive membrane properties of dendritic cables. We highlight the fact that in order to obtain selfconsistent traveling wave solutions it is necessary to have some dead-time immediately after firing. We then show how the solitary wave solutions obtained by Ermentrout (1998) can be recovered in the long-wavelength limit $k \rightarrow 0$ when $I_{0}<h$. That is, starting from a traveling wave solution, we show that an excitable network can support the propagation of solitary pulses whose speed is given by $c_{0}=\lim _{k \rightarrow 0} c(k)$. This is analogous to the long-wavelength limit analyzed by Ermentrout and Rinzel (1981) in their study of waves in a one-dimensional reactiondiffusion equation. The continuation of solitary wave solutions to finite periodic waves leads to a familiy of approximately velocity-independent solutions. We also establish that for fast solitary waves the velocity scales as some power of the synaptic strength $g$ with $c_{0} \sim \sqrt{g}$ for synaptic delays and $c_{0} \sim g^{2}$ for dendritic delays. This suggests that the approximately linear relationship observed experimentally (Golomb and Amitai 1997) might arise from some combination of these two aspects.

The stability of traveling waves is then analyzed in section 3 by considering perturbations of the firing times. First, a linearized firing time map is constructed in the form of a linear integrodifference equation of infinite order. It is shown how the condition for asymptotic stability can be expressed in terms of the solutions of an associated characteristic equation. Taking the longwavelength limit we then study the stability of solitary pulses in the excitable regime (section 4). In particular, we show that solitary wave solutions typically come in pairs with the faster (slower) solution stable (unstable) in the case of zero axonal delays; for non-zero delays and fast synapses the stable wave can itself destabilize via a Hopf bifurcation. We end in section 5 with a brief discussion of waves in two-dimensional networks and relate our stability analysis to recent work by Kistler et al (1998). In particular, we point out that their analysis is based on a weaker notion of stability than considered in this paper. 


\section{Traveling waves and pulses}

Consider a one-dimensional network of integrate-and-fire (IF) neurons with excitatory synaptic coupling evolving according to the equation

$$
\frac{\partial V(x, t)}{\partial t}=I_{0}-\frac{V(x, t)}{\tau_{m}}+I(x, t) .
$$

Here $V(x, t)$ denotes the membrane potential of the neuron at $x \in \mathbb{R}$ at time $t, \tau_{m}$ is the membrane time constant, $I_{0}$ is a constant external input, and $I(x, t)$ is the total synaptic input into the cell. Equation (2.1) is supplemented by the reset condition $V\left(x, t^{+}\right)=\zeta$ whenever $V(x, t)=h$, where $h$ is the threshold for firing. We shall set $h=1$ and $\tau_{m}=1$ for convenience. This fixes the units of time to be of the order $10 \mathrm{msec}$. In the absence of any synaptic inputs, we can distinguish two distinct operating regimes: (a) oscillatory $\left(I_{0}>1\right)$ - each neuron independently fires at regular intervals of period $T_{0}=\ln \left(\left[I_{0}-\zeta\right] /\left[I_{0}-1\right]\right)$, and (b) excitable $\left(I_{0}<1\right)$ - the neurons require an additional input before they can fire. In this paper we shall restrict ourselves mainly to the excitable regime, since we have studied oscillatory IF networks in some detail elsewhere (Bressloff and Coombes 1999). The synaptic current is taken to be of the form

$$
I(x, t)=g \int_{-\infty}^{\infty} \int_{0}^{\infty} W\left(x-x^{\prime}\right) J(\tau) E\left(x^{\prime}, t-\tau\right) d \tau d x^{\prime}
$$

where the strength of the interactions between cells is determined by the coupling parameter $g$, $g>0$, and the pattern of connectivity is specified by the positive weight function $W(x)$, which is assumed to be symmetric, $W(-x)=W(x)$, and a monotonically decreasing function of $|x|$ with $\int_{0}^{\infty} W(x) d x<\infty$. For concreteness, we take $W(x)$ to be the exponential function

$$
W(x)=\frac{1}{2 \sigma} \mathrm{e}^{-|x| / \sigma}
$$

with $\sigma$ characterizing the range of interactions. (The basic results of this paper do not depend on the precise form of $W(x)$ ). Neglecting the shape of an individual pulse, the output spike train of each oscillator is represented as a sequence of Dirac delta-functions,

$$
E(x, t)=\sum_{m=-\infty}^{\infty} \delta\left(t-T_{m}(x)\right)
$$

where $T_{m}(x)$ is the $m$ th firing-time of the oscillator at $x$, that is, $V\left(x, T_{m}(x)\right)=1$ for all integers $m$. Each incoming spike is converted to a post-synaptic potential whose shape is represented by the delay kernel $J(\tau)$ : various factors can contribute to $J(\tau)$ including axonal propagation delays, synaptic processing and dendritic processing (see below).

Following Bressloff and Coombes (1997), we define a traveling wave to be a self-consistent solution to equations (2.1)-(2.3) in which the activity $E(x, t)$ is of the particular form $E(x, t)=$ $\sum_{m \in \mathbb{Z}} \delta(t-(k x+m) T)$ for some finite period $T$. The speed of the wave is $c=(k T)^{-1}$ for a given wavenumber $k$. For such a solution, the neurons are firing at regular intervals of collective 
period $T$ such that $T_{m}(x)=m T+\theta(x) T$ with $\theta(x)=k x$ the firing phase of the neuron at $x$. Thus traveling wave solutions belong to the class of phase-locked solutions studied previously by Bressloff and Coombes (1999), and we can develop the analysis along similar lines. First, under the given ansatz for the activity $E(x, t)$, we integrate equation (2.1) between two successive firing times. Incorporating the reset condition then leads to the integral equation

$$
1-\mathrm{e}^{-T} \zeta=\left(1-\mathrm{e}^{-T}\right) I_{0}+g \int_{-\infty}^{\infty} W(x) K_{T}(k x) d x
$$

with

$$
K_{T}(\theta)=\mathrm{e}^{-T} \int_{0}^{T} \mathrm{e}^{\tau} \sum_{m=-\infty}^{\infty} J(\tau+(m-\theta) T) d \tau
$$

Since $K_{T}(\theta)$ is a real periodic function of $\theta$ it can be expanded as a Fourier series:

$$
K_{T}(\theta)=\frac{\left[1-\mathrm{e}^{-T}\right]}{T} \sum_{m}[a(2 \pi m / T) \cos (2 \pi m \theta)-b(2 \pi m / T) \sin (2 \pi m \theta)]
$$

where

$$
a(\omega)=\operatorname{Re} \frac{\widetilde{J}(\omega)}{1+i \omega}, b(\omega)=-\operatorname{Im} \frac{\widetilde{J}(\omega)}{1+i \omega}
$$

and $\widetilde{J}(\omega)$ is the Fourier transform of $J(\tau)$,

$$
\widetilde{J}(\omega)=\int_{-\infty}^{\infty} \mathrm{e}^{-i \omega \tau} J(\tau) d \tau
$$

such that

$$
J(\tau)=\int_{-\infty}^{\infty} \mathrm{e}^{i \omega \tau} \widetilde{J}(\omega) \frac{d \omega}{2 \pi} .
$$

Note that $\widetilde{J}(\omega)$ is analytic in the lower-half complex $\omega$-plane since $J(\tau)=0$ for $\tau \leq 0$. Substituting equation (2.7) into equation (2.5) and exploiting the fact that $W(x)$ is symmetric, we obtain the result

$$
1-\mathrm{e}^{-T} \zeta=\left(1-\mathrm{e}^{-T}\right) I_{0}+g \frac{1-\mathrm{e}^{-T}}{T} \sum_{m} a(2 \pi m / T) \widetilde{W}(2 \pi m k)
$$

where $\widetilde{W}(p)$ is the Fourier transform of $W(x)$. For the exponenential weight function (2.3) we have

$$
\widetilde{W}(p)=\frac{1}{1+\sigma^{2} p^{2}}
$$

Equation (2.11) generates a dispersion relation which we can write either as $c=c(k)$ or $\nu=\nu(k)$ where $\nu=T^{-1}$. 
An interesting question concerns what happens as $k \rightarrow 0$. Ermentrout and Rinzel (1981) have established that in the case of a one-dimensional reaction-diffusion equation, $c \rightarrow \infty$ as $k \rightarrow 0$ in the oscillatory regime whereas $c \rightarrow c_{0}<\infty$ as $k \rightarrow 0$ in the excitatory regime. In other words, only the latter supports the propagation of a solitary traveling wave or pulse, with $c_{0}$ the speed of the pulse. A similar result holds for the IF model with excitatory coupling $(g>0)$. This is most easily seen by taking the limit $T \rightarrow \infty$ in equation (2.5) with $K_{T}(\theta)$ given by equation (2.6). First note that equation (2.5) only has solutions for large $T$ if $I_{0}<1$ (excitable regime). Without loss of generality, we set $I_{0}=0$. Second, $K_{T}(k x) \approx \int_{-T}^{0} \mathrm{e}^{t} J(t-k x T) d t$ for large $T$ so that $K_{T}(k x) \rightarrow 0$ as $T \rightarrow \infty$ unless $k=\left(c_{0} T\right)^{-1}$ for some $T$-independent constant speed $c_{0}$. (It should be noted that there also exists a finite- $T$ solution in the limit $k \rightarrow 0$, which corresponds to the synchronous solution). Taking the large- $T$ limit of equation (2.5) then yields the result

$$
\begin{aligned}
1 & =g \int_{-\infty}^{\infty} W(x) \int_{-\infty}^{0} \mathrm{e}^{t} J\left(t-x / c_{0}\right) d t d x \\
& =g \int_{0}^{\infty} W(x) \mathrm{e}^{-x / c_{0}} \int_{0}^{x / c_{0}} \mathrm{e}^{t} J(t) d t d x
\end{aligned}
$$

where we have used the fact that $J(t)=0$ for $t \leq 0$. Equation (2.13) is identical to the selfconsistency condition for a solitary pulse $E(x, t)=\delta\left(t-x / c_{0}\right)$ obtained previously by Ermentrout (1998). From this equation, scaling laws can be derived for the dependence of the velocity $c_{0}$ on various parameters such as the coupling strength $g$ and the range of interactions $\sigma$ of $W(x)$ (Ermentrout 1998). A simple method for evaluating the integrals in equation (2.13) is to use the Fourier transform of $J(\tau)$. Substituting equations (2.10) and (2.3) into (2.13) and performing the integrations over $x$ and $t$ we obtain the result

$$
1=\frac{g c_{0}}{2} \int_{-\infty}^{\infty} \frac{\widetilde{J}(\omega)}{1+i \omega}\left[\frac{1}{c_{0}-i \omega}-\frac{1}{1+c_{0}}\right] \frac{d \omega}{2 \pi}
$$

where we have rescaled $c_{0}$ according to $c_{0} \rightarrow c_{0} / \sigma$. Closing the contour in the lower-half complex plane then picks up the pole at $\omega=-i c_{0}$ :

$$
1=\frac{g c_{0}}{2\left(1+c_{0}\right)} \widetilde{J}\left(-i c_{0}\right) .
$$

\subsection{Synaptic delay kernel}

We shall now illustrate our analysis of wave propagation in IF networks for particular choices of $J(\tau)$. One important contribution to the finite-impulse response of a neuron occurs at the synapses. An incoming spike due to the firing of a pre-synaptic neuron causes the release of neurotransmitters that diffuse across the synaptic gap and bind to receptors in the cell membrane of the post-synaptic neuron. This induces a time-dependent change in the membrane conductance that may be modelled as a second-order Markov scheme leading to the so-called alpha function response (Destexhe et al 1994). Combining this with the effects of discrete delays due to the 


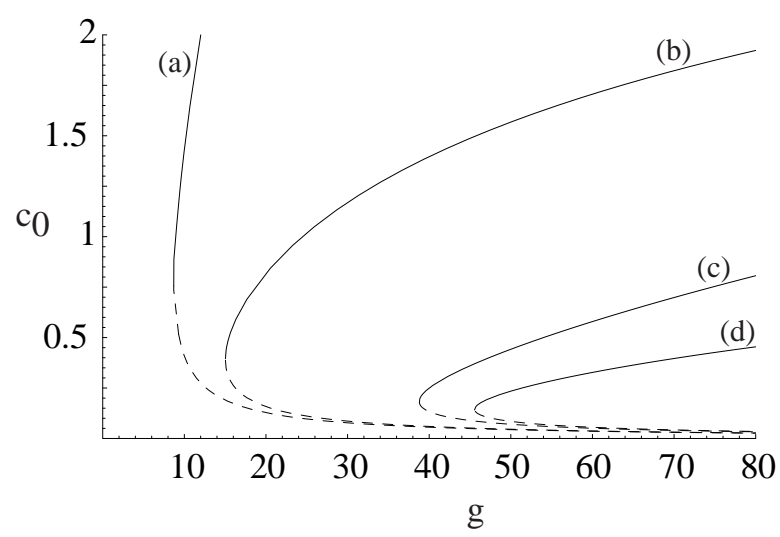

Figure 1: Variation of the speed $c_{0}$ of a traveling pulse as a function of the synaptic coupling $g$ for different values of the inverse rise-time $\alpha$ and axonal delay $\tau_{a}$. (a) $\alpha=2, \tau_{a}=0$, (b) $\alpha=2, \tau_{a}=1$, (c) $\alpha=0.25, \tau_{a}=0$, (d) $\alpha=0.25, \tau_{a}=1$. Solid (dashed) lines correspond to stable (unstable) branches (see section 4). The external input is taken to be $I_{0}=0$ and $\sigma=1$.

finite propagation time of spikes along the axons, we introduce a synaptic delay kernel of the form

$$
J(\tau)=\alpha^{2}\left(\tau-\tau_{a}\right) \mathrm{e}^{-\alpha\left(\tau-\tau_{a}\right)} \Theta\left(\tau-\tau_{a}\right)
$$

where $\alpha$ is the inverse rise-time for synaptic response, $\tau_{a}$ is an axonal propagation delay, and $\Theta(\tau)=1$ if $\tau>0$ and is zero otherwise. Substitution of equation (2.16) into (2.15) leads to the following implicit equation for the propagation velocity of a solitary pulse:

$$
1=\frac{g \alpha^{2} c_{0}}{2\left(1+c_{0}\right)} \frac{\mathrm{e}^{-c_{0} \tau_{a}}}{\left(\alpha+c_{0}\right)^{2}}
$$

The case $\tau_{a}=0$ was previously studied by Ermentrout (1998), who highlighted the fact that for large $c_{0}$, the velocity scales according to a power law $c_{0} \sim \alpha \sigma \sqrt{g / 2}$. This form of power law is consistent with the behaviour observed in more detailed computational models (Golomb and Amitai 1997). The velocity $c_{0}$ is plotted as a function of the coupling $g$ in figure 1 . It can be seen that for fixed $\alpha$ and $\tau_{a}$ there exists a critical coupling $g_{s}\left(\alpha, \tau_{a}\right)$ such that there are no traveling pulse solutions for $g<g_{s}$ and two solutions for $g>g_{s}$. We shall establish in section 4 that for the given parameter values, the lower (upper) solution branch is unstable (stable).

Let us now turn to the case of traveling wave solutions with finite $T$. For the synaptic delay kernel (2.16), equation (2.8) becomes

$$
\begin{aligned}
& \frac{a(\omega)}{\alpha^{2}}=\frac{\left(\alpha^{2}-\omega^{2}-2 \omega^{2} \alpha\right) \cos \left(\omega \tau_{a}\right)-\left(\omega\left(\alpha^{2}-\omega^{2}\right)+2 \omega \alpha\right) \sin \left(\omega \tau_{a}\right)}{\left(\alpha^{2}+\omega^{2}\right)^{2}\left(1+\omega^{2}\right)}, \\
& \frac{b(\omega)}{\alpha^{2}}=\frac{\left(\omega\left(\alpha^{2}-\omega^{2}\right)+2 \omega \alpha\right) \cos \left(\omega \tau_{a}\right)+\left(\alpha^{2}-\omega^{2}-2 \omega^{2} \alpha\right) \sin \left(\omega \tau_{a}\right)}{\left(\alpha^{2}+\omega^{2}\right)^{2}\left(1+\omega^{2}\right)} .
\end{aligned}
$$




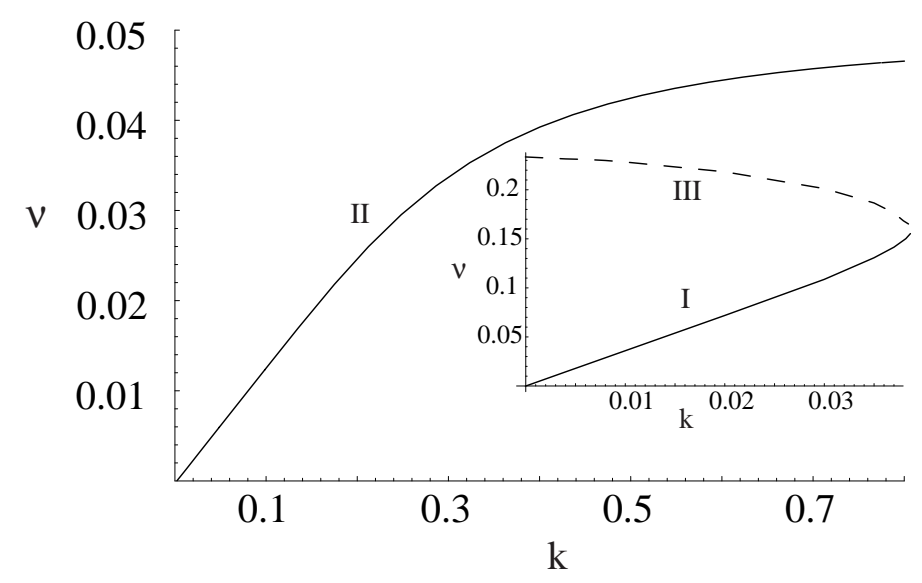

Figure 2: Dispersion curves showing how the frequency $\nu=T^{-1}$ of a traveling wave varies with the wavenumber $k$ for $\alpha=2, \tau_{a}=0, I_{0}=0, \zeta=0$ and $g=20>g_{s}$. Curves I and II are continuations of the long-wavelength solutions $\nu=c_{0} k$ with $c_{0}$ satisfying equation (2.17), see fig. 1. Curve III is the continuation from the synchronous solution at $k=0$ (see inset).

Solving equation (2.11) using (2.12) and (2.18), we calculate dispersion curves $\nu=\nu(k)$ for finite wavelength traveling waves. Typical results in the excitable regime for $g>g_{s}$ and fixed $\alpha$ and $\tau_{a}$ are shown in figure 2. It can be seen that there are three dispersion curves labelled I-III. Curves I and II are continuations of the long-wavelength finite velocity solutions displayed in figure 1 , whereas the third is a continuation of the synchronous solution. Both curves I and II are approximately linear in $k$, at least for sufficiently long wavelengths. Hence there exist families of approximately velocity-independent solutions, which is consistent with the observations of multiple discharge patterns (Golomb and Amitai 1997). Curves I and III only exist for small values of $k$ and annihilate each other in a saddle-node bifurcation at a critical value of $k$. These results should be contrasted with the case $g<g_{s}$ for which there are no long-wavelength solitary pulses and hence only one dispersion curve. The continuation of the synchronous solution now persists into the large- $k$ regime. The stability of traveling waves is discussed in section 3 .

\subsection{Dendritic delay kernel}

It is well known that the passive membrane properties of a neuron's dendritic tree result in a diffusive spread of current through the system that induces changes in the membrane potential along the tree. This diffusive process can be modeled in terms of a second-order linear partial differential equation known as the cable equation (Rall 1989). For the sake of concreteness, we shall take each neuron to consist of a soma or cell body where spike generation occurs, which is connected resistively to the end of a semi-infinite uniform dendritic cable. As a further simplification, suppose that all synapses are located at a fixed position $\xi_{0}, 0 \leq \xi_{0}<\infty$ on 


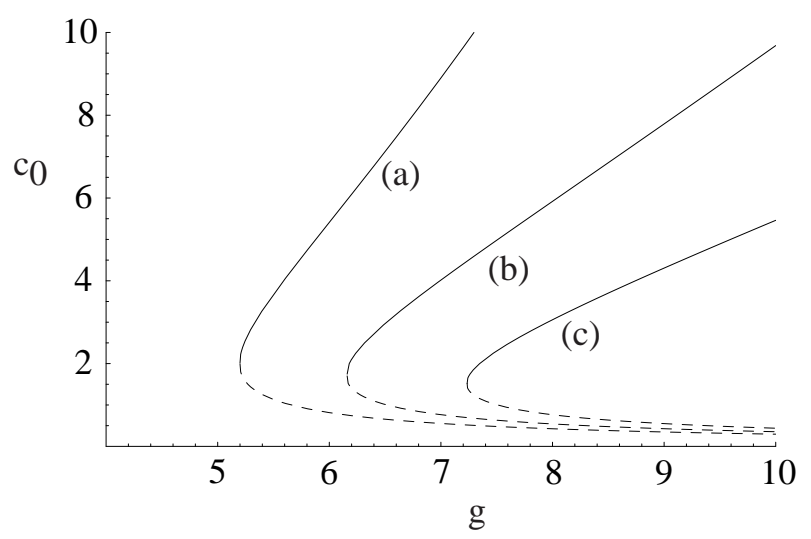

Figure 3: Variation of the speed $c_{0}$ of a traveling pulse as a function of the synaptic coupling $g$ for different values of the dendritic coordinate $\xi_{0}$. (a) $\xi_{0}=0$, (b) $\xi_{0}=0.1$, (c) $\xi_{0}=0.2$. We have set $D=\tau_{d}=\sigma=1$. Solid (dashed) curves are stable (unstable), see section 4 .

the dendritic cable independently of the positions of the interacting neurons. Solving the cable equation then leads to an effective synaptic current of the form $(2.2)$ with $J(\tau)=G\left(\xi_{0}, \tau\right)$ and (Bressloff 1999)

$$
G(\xi, t)=\frac{\mathrm{e}^{-t / \tau_{d}}}{\sqrt{\pi D t}} \mathrm{e}^{-\xi^{2} / 4 D t} \Theta(t)
$$

where $\tau_{d}$ is the membrane time constant of the cable and $D$ is the diffusivity. The Green's function $G(\xi, t)$ determines the membrane potential response at the end of the cable due to an instantaneous injection of unit current at point $\xi$ at time $t$. As in the case of synaptic delays, it is useful to consider the Fourier transform of the delay kernel, $\widetilde{J}(\omega)=\widetilde{G}\left(\xi_{0}, \omega\right)$ where

$$
\widetilde{G}(\xi, \omega)=\frac{1}{D \gamma(\omega)} \mathrm{e}^{-\gamma(\omega) \xi}
$$

is the transfer function of the dendritic cable with

$$
\gamma(\omega)^{2}=\frac{1+i \omega \tau_{d}}{D \tau_{d}}
$$

Note that $\gamma(\omega)$ in equation (2.21) is related to the membrane impedance of the dendritic cable.

We can use equation (2.21) to investigate how the presence of passive dendritic interactions influences wave propagation in a one-dimensional network of IF neurons. We first consider the propagation of single pulses in the long-wavelength limit. The allowed velocities $c_{0}$ of these pulses are given by solutions of equation $(2.15)$ with $\widetilde{J}(\omega)=\widetilde{G}\left(\xi_{0}, \omega\right)$. That is, setting $\tau_{d}=1, D=1$ we have

$$
1=\frac{g c_{0}}{2\left(1+c_{0}\right)} \frac{\mathrm{e}^{-\xi_{0} \sqrt{1+c_{0}}}}{\sqrt{1+c_{0}}}
$$




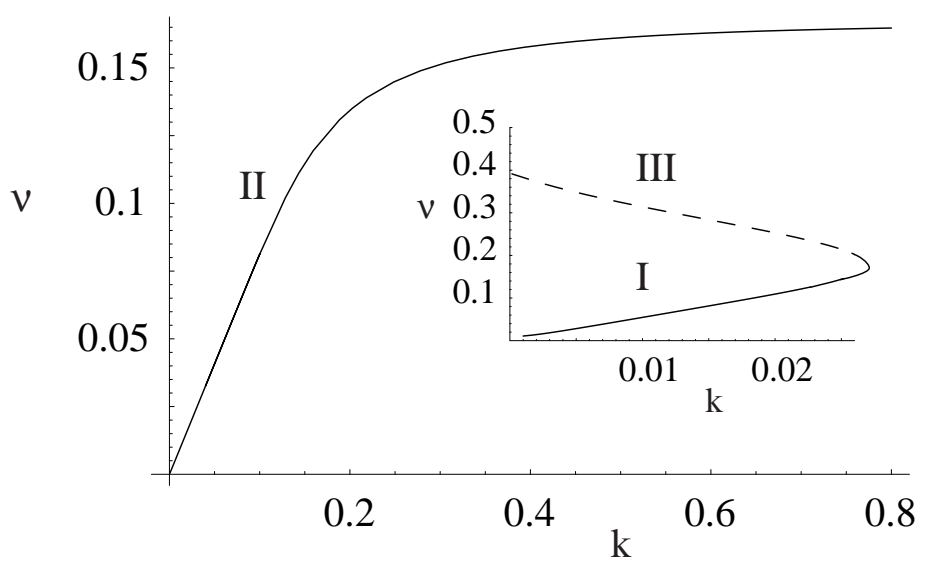

Figure 4: Dispersion curves showing how the frequency $\nu=T^{-1}$ of a traveling wave varies with the wavenumber $k$ for a dendritic delay kernel with $\xi_{0}=0, I_{0}=0, \zeta=0$ and $g=6>g_{s}$. Curves I and II are continuations of the long-wavelength solutions $\nu=c_{0} k$ with $c_{0}$ satisfying equation (2.23), see fig. 3. Curve III is the continuation from the synchronous solution at $k=0$ (see inset).

We plot solutions $c_{0}(g)$ for various values of $\xi_{0}$ in figure 3 . The qualitative behaviour is similar to that found for synaptic delays, see figure 1 . However, for synapses proximal to the soma $\left(\xi_{0}=0\right)$, we have a power scaling law given by $c_{0} \sim g^{2}$, rather than $c_{0} \sim \sqrt{g}$ as found for synaptic delays. Recent experimental studies of solitary wave propagation in cortical slices show that for fast waves the velocity appears to increase approximately linearly with synaptic strength (Golomb and Amitai 1997), which lies somewhere between these two cases. Whether or not, the linear relationship arises from some combination of dendritic and synaptic delays, or is a consequence of more detailed membrane properties remains to be seen.

In the case of a traveling wave of non-zero wavenumber $k$, the frequency $\nu=T^{-1}$ is determined by the phase-locking equation (2.5) with the interaction kernel $K_{T}(\theta)$ having the Fourier expansion defined by equations (2.7) and (2.8). Evaluating the functions $a(\omega)$ and $b(\omega)$ for $\widetilde{J}(\omega)=\widetilde{G}\left(\xi_{0}, \omega\right)$ gives

$$
\begin{aligned}
& a(\omega)=\frac{[\bar{a}(\omega)-\omega \bar{b}(\omega)] \cos (\bar{b}(\omega) \xi)-[\omega \bar{a}(\omega)+\bar{b}(\omega)] \sin (\bar{b}(\omega) \xi)}{\left(1+\omega^{2}\right)\left(\bar{a}(\omega)^{2}+\bar{b}(\omega)^{2}\right)} \mathrm{e}^{-\bar{a}(\omega) \xi_{0}} \\
& b(\omega)=\frac{[\bar{a}(\omega)-\omega \bar{b}(\omega)] \sin (\bar{b}(\omega) \xi)+[\omega \bar{a}(\omega)+\bar{b}(\omega)] \cos (\bar{b}(\omega) \xi)}{\left(1+\omega^{2}\right)\left(\bar{a}(\omega)^{2}+\bar{b}(\omega)^{2}\right)} \mathrm{e}^{-\bar{a}(\omega) \xi_{0}}
\end{aligned}
$$

where

$$
\bar{a}(\omega)=\frac{1}{\sqrt{D \tau_{d}}} \sqrt{\frac{1}{2}\left[1+\sqrt{1+\left(\tau_{d} \omega\right)^{2}}\right]}
$$




$$
\bar{b}(\omega)=\frac{\operatorname{sign}(\omega)}{\sqrt{D \tau_{d}}} \sqrt{\frac{1}{2}\left[-1+\sqrt{1+\left(\tau_{d} \omega\right)^{2}}\right]}
$$

and $\sqrt{D \tau_{d}}$ is the membrane space constant of the dendritic cable. Finally, we look for solutions of equation (2.11) using (2.12) and (2.24) in order to calculate dispersion curves $\nu=\nu(k)$ for finite wavelength traveling waves. Typical results in the excitable regime with $I_{0}=0$ and $g>g_{s}$ are shown in figure 4.

\subsection{Existence of traveling waves and re-excitation}

In the construction of the traveling wave dispersion curves of figures 2 and 4 we set the reset level to be zero $(\zeta=0)$ and searched for solutions of equation (2.11) for the collective period $T$ as a function of wavenumber $k$. However, considerable care has to be taken in the interpretation of these results. Let us consider a particular neuron in the network that is driven by a synaptic input of the form (2.2) under the assumption that all other neurons are phase-locked according to a traveling wave of wavenumber $k$ and period $T$. Suppose that the given neuron last fired at time $t=0$. Taking $T$ to be a solution of equation (2.11) simply means that the chosen neuron crosses threshold at time $T$. This need not lead to a self-consistent traveling wave solution since it is possible that the neuron has previously fired one or more times in the interval $(0, T)$. This is indeed found to be the case as illustrated in figure $5 \mathrm{a}$, where we plot $V(t)$ for $t>0$ assuming that the neuron evolves according to equation (2.1) with synaptic delays (and subsequent resetting of the neuron suppressed). More explicitly,

$$
\begin{aligned}
V(t)= & -\mathrm{e}^{-t} \zeta+\left(1-\mathrm{e}^{-t}\right) I_{0} \\
& +\frac{g}{T} \sum_{n}\left(a(2 \pi n / T)\left[\cos (2 \pi n t / T)-\mathrm{e}^{-t}\right]+b(2 \pi n / T) \sin (2 \pi n t / T)\right) \widetilde{W}(2 \pi n k)
\end{aligned}
$$

with $a(\omega), b(\omega)$ defined by equations (2.18) and (2.19). It can be seen from figure 5a that although the potential does cross the threshold $h=1$ at time $T$, there is a rapid re-excitation at a time $0<t \ll T$ and this yields an inconsistency. In order to generate a self-consistent solution it is necessary to introduce some form of dead-time immediately after firing either through a time-varying threshold or, as shown in figure 5b, through a negative reset level $\zeta \ll 0$. When this is combined with subsequent resetting a self-consistent traveling wave of period $T$ is obtained.

In the long wave-length limit, the issue of fast re-excitation is no longer relevant as far as the existence of a solitary wave is concerned. However, it does have practical relevance when attempting to numerically simulate the propagation of solitary pulses. As discussed by Ermentrout (1998), there appears to be a complex series of transitions from single spike events to states with repetitive firing. We hope to explore this particular issue further elsewhere. 

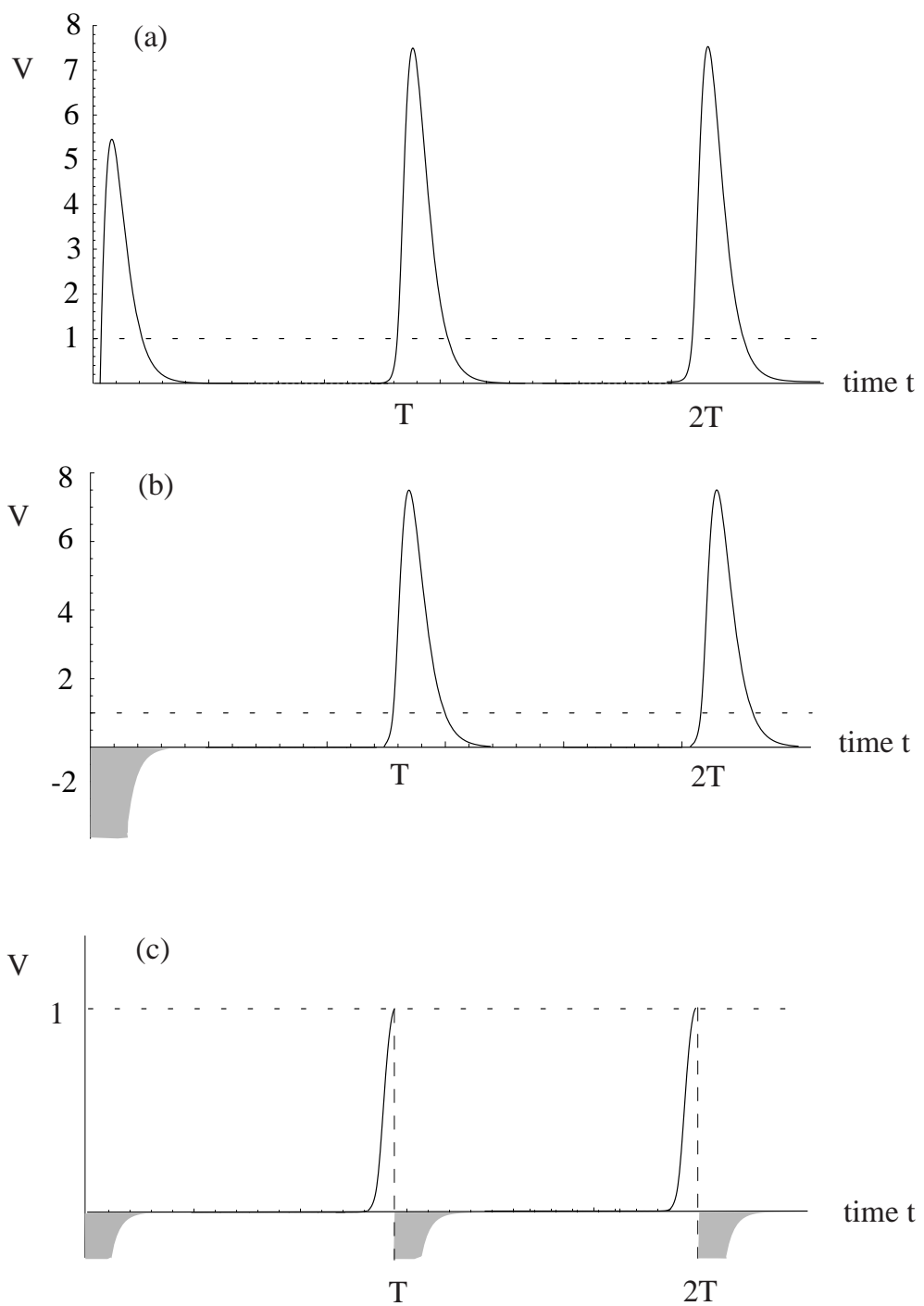

Figure 5: The role of dead-time in the construction of a self-consistent traveling wave solution for $\alpha=2, \tau_{a}=0, I_{0}=0, g=20$ and wavenumber $k=0.01$. The corresponding collective period is $T \approx 27.8$ (see inset of fig. 2). A given neuron is assumed to fire at time $t=0$ and its potential $V(t)$ is plotted for $t>0$. (a) Evolution of $V$ without dead-time $(\zeta=0)$ and no subsequent reset. (b) Evolution of $V$ with non-zero dead-time $(\zeta \ll 0)$ and no subsequent reset. (c) Evolution of $V$ with non-zero dead-time and subsequent reset. 


\section{$3 \quad$ Stability analysis and the linearized firing time map}

In this section we shall analyze the stability of traveling waves in a one-dimensional network of IF neurons by considering perturbations of the firing times. We shall find that these perturbations evolve according to a nonlinear firing time map, which is in the form of an integro-difference equation of infinite order. Conditions for the local asymptotic stability of a given traveling wave can then be expressed in terms of the solutions of a characteristic equation obtained by linearizing this firing time map. This can be established by introducing a generalized $z$-transform (discrete Laplace transform) that takes into account the infinite-dimensional nature of the IF network dynamics and the fact that we are interested in convective instabilities in the case of traveling waves. We shall ignore the effects of dead-time in the following calculation, which is a reasonable approximation for long-wavelength traveling waves since the period $T$ is large.

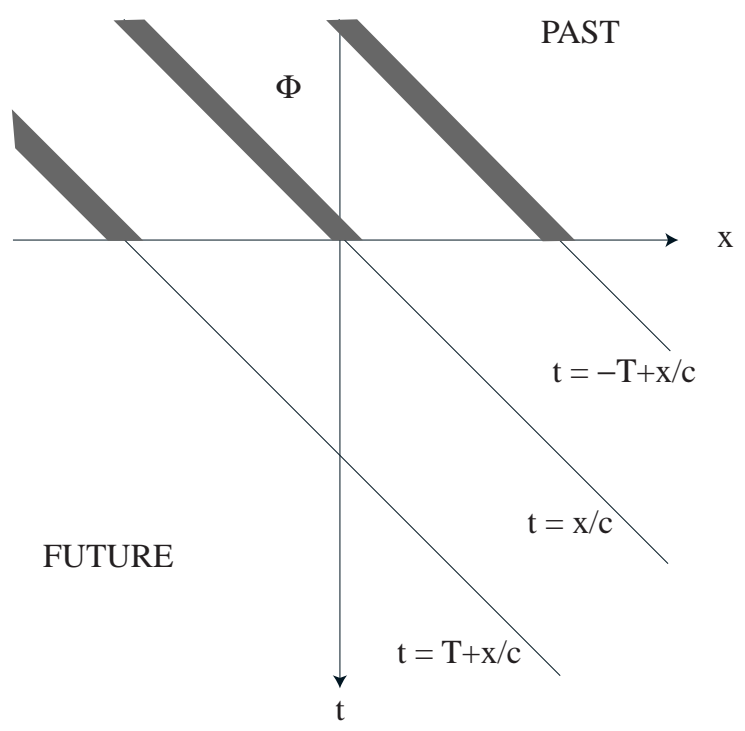

Figure 6: The Cauchy problem for the linearized firing time map (3.2). Successive wavefronts are shown with speed $c=1 / k T$. The initial data $\Phi$ specifies the initial perturbations of the firing times defined along the wavefronts in the past, $n+k x \leq 0$. The traveling wave is asymptotically stable if these (non-uniform) perturbations decay to zero as $t \rightarrow \infty$, that is, $u_{n}(x) \rightarrow 0$ as $n+k x \rightarrow \infty$.

Following along similar lines to our previous work (Bressloff and Coombes 1999), we first integrate equation (2.1) from $T_{n-1}(x)$ to $T_{n}(x)$ using the reset condition to obtain the nonlinear firing time map

$$
\begin{aligned}
\mathrm{e}^{T_{n}(x)}= & I_{0}\left[\mathrm{e}^{T_{n}(x)}-\mathrm{e}^{T_{n-1}(x)}\right] \\
& +g \int_{T_{n-1}(x)}^{T_{n}(x)} \mathrm{e}^{t} \int_{-\infty}^{\infty} W\left(x-x^{\prime}\right) \sum_{m \in \mathbb{Z}} J\left(t-T_{m}\left(x^{\prime}\right)\right) d x^{\prime} d t .
\end{aligned}
$$


We then set $T_{n}(x)=(n+k x) T+u_{n}(x)$ and expand the resulting map as a power series in the perturbations $u_{m}(x)$. To $\mathcal{O}(1)$ we recover equation (2.6) for the traveling wave state, whereas the $\mathcal{O}(u)$ term leads to a linear integro-difference equation of the form

$$
\begin{aligned}
\left(I_{0}-1+g A\right) u_{n}(x)= & \left(I_{0}-1+g[A-\bar{B}]\right) u_{n-1}(x) \\
& +g \sum_{m \in \mathbb{Z}} \int_{-\infty}^{\infty} B_{m-1}\left(x-x^{\prime}\right) u_{n-m}\left(x^{\prime}\right) d x^{\prime}
\end{aligned}
$$

with

$$
\begin{gathered}
A=\int_{-\infty}^{\infty} W(x) \sum_{m \in \mathbb{Z}} J([m+k x] T) d x, \\
B_{m}(x)=W(x) \mathrm{e}^{-T} \int_{0}^{T} \mathrm{e}^{t} J^{\prime}(t+[m+k x] T) d t,
\end{gathered}
$$

and

$$
\bar{B}=\sum_{m \in \mathbb{Z}} \int_{-\infty}^{\infty} B_{m}(x) d x .
$$

Note that $A, B_{m}(x)$ and $\bar{B}$ all depend on the self-consistent collective period $T$ and the wavenumber $k$. From equation (3.4), we see that $B_{m}(x)=0$ for $m+k x \leq-1$, which ensures that causality is not violated. This also suggests how to set up initial data for the system (see figure 6 ). Let $\mathcal{D}_{-}=\{(x, n) \mid x \in \mathbb{R}, n \in \mathbb{Z}, n+k x \leq 0\}$. Suppose that $u_{n}(x)=\phi_{n}(x)$ for $(x, n) \in \mathcal{D}_{\text {- where }}$ $\phi_{n}(x)$ is some bounded function on $\mathcal{D}_{-}, \sup \left\{\left|\phi_{n}(x)\right| \mid(x, n) \in \mathcal{D}_{-}\right\}<\infty$. We then decompose the solution as

$$
u_{n}(x ; \Phi)=v_{n}(x) \Theta(n+k x)+\phi_{n}(x) \bar{\Theta}(n+k x)
$$

with $\bar{\Theta}(x)=1-\Theta(x)$. Substitution of equation (3.6) into (3.2) shows that

$$
\begin{aligned}
& \left(I_{0}-1+g A\right) v_{n}(x) \\
& =\left(I_{0}-1+g[A-\bar{B}]\right) v_{n-1}(x) \Theta(n+k x-1) \\
& +g \sum_{m \in \mathbb{Z}} \int_{-\infty}^{\infty} B_{m-1}\left(x-x^{\prime}\right) v_{n-m}\left(x^{\prime}\right) \Theta\left(n-m+k x^{\prime}\right) d x^{\prime}+F_{n}^{\phi}(x)
\end{aligned}
$$

for $n+k x>0$, where

$$
\begin{aligned}
F_{n}^{\phi}(x)= & \left(I_{0}+g A-g \bar{B}\right) \phi_{n-1}(x) \bar{\Theta}(n+k x-1) \\
& +g \sum_{m \in \mathbb{Z}} \int_{-\infty}^{\infty} B_{m-1}\left(x-x^{\prime}\right) \phi_{n-m}\left(x^{\prime}\right) \bar{\Theta}\left(n-m+k x^{\prime}\right) d x^{\prime} .
\end{aligned}
$$

In order to solve the inhomogeneous linear equation (3.7), we introduce the double transform $\mathcal{F}(u)$ according to

$$
\mathcal{F}(u)(z, p)=\sum_{n \in \mathbb{Z}} \int_{-\infty}^{\infty} \mathrm{e}^{-i p x} z^{-(n+k x)} \Theta(n+k x) u_{n}(x) d x .
$$


We shall assume that the solutions $u_{n}(x)$ are exponentially bounded in the sense that $u_{n}(x)<$ $\kappa r^{n+k x}$ for $n+k x>0$ and constants $\kappa, r$. The transform (3.9) is a combination of a $z$-transform (discrete Laplace transform) and a Fourier transform. Note that the time-like variable $z$ scales as $z^{-(n+k x)}$, since we are interested in studying convective instabilities in the moving frame of the traveling wave. Let us apply the double transform (3.9) to equation (3.7):

$$
\begin{aligned}
z\left(I_{0}-1+g A\right) \widetilde{v}(z, p)= & \left(I_{0}-1+g[A-\bar{B}]\right) \widetilde{v}(z, p) \\
& +\widetilde{B}(z, p) \widetilde{v}(z, p)+z \widetilde{F}^{\Phi}(z, p)
\end{aligned}
$$

where $\widetilde{F}^{\Phi}=\mathcal{F}\left(F^{\Phi}\right)$ and

$$
\widetilde{B}(z, p)=\sum_{n \in \mathbb{Z}} \int_{-\infty}^{\infty} \mathrm{e}^{-i p x} z^{-(n+k x)} B_{n}(x) d x
$$

After a rearrangement we find that

$$
\widetilde{v}(z, p)=\widetilde{X}(z, p) \widetilde{F}^{\Phi}(z, p),
$$

with $\widetilde{X}(z, p)=\frac{z}{\widetilde{H}(z, p)}$ and

$$
\widetilde{H}(z, p)=(1-z)\left[I_{0}-1+g A\right]+g[\widetilde{B}(z, p)-\widetilde{B}(1,0)] .
$$

We shall now establish that the long-time behavior of $u_{n}(x)$ is governed by the zeros of $\widetilde{H}(z, p)$, that is, by solutions to the characteristic equation $\widetilde{H}(z, p)=0$. First note that one solution to this equation is $z=1$ at $p=0$. This reflects invariance of the system under uniform phase-shifts $T_{n}(x) \rightarrow T_{n}(x)+\delta$. We shall exclude this case by requiring that the initial data $\Phi$ involves non-uniform perturbations. If we define

$$
r_{0}=\sup \{|z| \mid \widetilde{H}(z, p)=0, p \in \mathbb{R}, z \neq 1\},
$$

then we can introduce the inverse double transform

$$
X_{n}(x)=\mathcal{F}^{-1}(\widetilde{X})(z, p) \equiv \oint_{C} \frac{d z}{2 \pi i} \int_{-\infty}^{\infty} \frac{d p}{2 \pi} \mathrm{e}^{i p x} \frac{z^{n+k x}}{\widetilde{H}(z, p)}
$$

where $C=\left\{z \in \mathbb{C}|| z \mid>\min \left\{r_{0}, 1\right\}\right\}$ and $n+k x>0$. We also define the convolution $u * v$ of two exponentially bounded sets of functions $u_{n}(x), v_{n}(x)$, integer $n$, to be

$$
\begin{aligned}
{[u * v]_{n}(x)=} & \sum_{m \in \mathbb{Z}} \int_{-\infty}^{\infty} u_{m}\left(x-x^{\prime}\right) v_{n-m}\left(x^{\prime}\right) \\
& \times \Theta\left(m+k\left(x-x^{\prime}\right)\right) \Theta\left(n-m+k x^{\prime}\right) d x^{\prime}
\end{aligned}
$$

such that $\mathcal{F}(u * v)=\mathcal{F}(u) \mathcal{F}(v)$. We can then use this convolution theorem for $\mathcal{F}$ to invert equation (3.12) and thus obtain the solution

$$
u_{n}(x ; \Phi)=\left[X * F^{\Phi}\right]_{n}(x)
$$


for $n+k x>0$. It follows from equations (3.14), (3.15), (3.17) and the boundedness of the initial data $\phi_{n}(x)$ that (for non-uniform initial data)

$$
\left|u_{n}(x ; \Phi)\right|<K r^{n+k x}, \quad n+k x>0, \quad r>r_{0} .
$$

Hence, $\lim _{n \rightarrow \infty} u_{n}(x ; \Phi)=0$ provided that all but one of the zeros of $\widetilde{H}(z, p)$ lie inside the unit circle.

Returning to the nonlinear firing time map (3.1), we can collect together the above results in the form of a stability theorem:

Theorem 1 Consider a traveling wave solution of wavenumber $k$ and collective period $T$ satisfying the phase-locking equation (2.5). Suppose that we decompose the set of firing times $\mathcal{T}$ of an IF network according to $\mathcal{T}^{ \pm}=\left\{T_{m}(x),(x, m) \in \mathcal{D}_{ \pm}\right\}$where $\mathcal{D}_{+}=\{(x, m), x \in \mathbb{R}, m \in \mathbb{Z} \mid m+k x>$ $0\}$ and $\mathcal{D}_{-}=\{(x, m), x \in \mathbb{R}, m \in \mathbb{Z} \mid m+k x \leq 0\}$. Let $\mathcal{T}^{+}$represent the solution to the Cauchy problem specified by the initial data $\mathcal{T}^{-}$(see figure 6$)$. Set $\phi_{m}(x)=T_{m}(x)-(m+k x) T$. We shall say that the traveling wave solution is (locally) stable iff for all $\epsilon>0$ there exists $\delta>0$ such that if $\sup \left\{\left|\phi_{m}(x)\right|,(x, m) \in \mathcal{D}_{-}\right\}<\delta$ then $\sup \left\{\left|\phi_{m}(x)\right|,(x, m) \in \mathcal{D}_{+}\right\}<\epsilon$. The solution is said to be asymptotically stable if it is locally stable and there exists $\delta>0$ such that if $\sup \left\{\left|\phi_{m}(x)\right|,(x, m) \in \mathcal{D}_{-}\right\}<\delta$ then $\lim _{n \rightarrow \infty} \sup \left\{\left|\phi_{n}(x)\right|, x \in \mathbb{R}\right\}=0$. (We exclude uniform initial data with respect to which a solution is always neutrally stable). We then have the following stability result: a traveling wave solution is (locally) asymptotically stable if the solutions of the characteristic equation

$$
(z-1)\left[I_{0}-1+g A\right]=g[\widetilde{B}(z, p)-\widetilde{B}(1,0)],
$$

satisfy $|z|<1$ for all $p \in \mathbb{R}$ (except for one solution $z=1$ at $p=0$ ). The coefficient $A$ is defined by equation (3.3) whilst $\widetilde{B}(z, p)$ is given by equations (3.11) and (3.4).

Note that the characteristic equations (3.19) has an infinite number of solutions for $z$. There are two distinct reasons for this. First, we are representing neural tissue as a one-dimensional continuum so that the linearized firing time map (3.2) has a continuous spectrum. (There is no discrete spectrum for this particular system). This is reflected by the fact that the solutions $z$ depend on the real variable $p$. Second, for a given $p$, there may still be an infinite (but countable) set of solutions due to the fact that the linear map (3.2) is of infinite-order with respect to the discrete time index $n$. Therefore, in general, it is a non-trivial problem determining the stability of a traveling wave solution. However, considerable simplification occurs in the long-wavelength limit in the excitable regime, which we shall discuss in section 4 . We end this section by briefly discussing another useful limiting case, namely, weak coupling in the oscillatory regime.

The stability of networks of IF neurons operating in the oscillatory regime has been studied extensively elsewhere so we shall only briefly describe some of the main results here (Bressloff and Coombes 1998a-c,1999). For $I_{0}>1$ and sufficiently small $g$, solutions to equation (3.19) 
in the complex $z$-plane will either be in a neighborhood of the real solution $z=1$ or in a neighborhood of one of the poles of $\widetilde{B}(z, p)$. It can be shown that the latter all lie inside the unit circle so that the stability of the traveling wave state will be determined by expanding (3.19) in powers of $g$ such that $z=1+\mathcal{O}(g)$ :

$$
(z-1)\left(I_{0}-1\right)=\left.g[\widetilde{B}(1, p)-\widetilde{B}(1,0)]\right|_{T=T_{0}}+\mathcal{O}\left(g^{2}\right)
$$

with $T_{0}=\ln \left[\left(I_{0} /\left(I_{0}-1\right)\right]\right.$. Comparison of equations (3.4) and (3.11) with the definition of the interaction function $K_{T}(\theta)$ in equation (2.6) shows that to first order in $g$, equation (3.20) reduces to

$$
(z-1)\left(I_{0}-1\right)=g \int_{-\infty}^{\infty} W(x)\left[\mathrm{e}^{-i p x}-1\right] K_{T_{0}}^{\prime}(k x) d x .
$$

Hence, the traveling wave will be asymptotically stable if all solutions of $(3.21)$ satisfy $\operatorname{Re} z(p)<0$ for all $p \neq 0$. For the sake of illustration, consider the particular case of the synchronous solution $k=0$. The condition for asymptotic stability then reduces to

$$
[\widetilde{W}(p)-\widetilde{W}(0)] K_{T_{0}}^{\prime}(0)<0
$$

for all $p \neq 0$. Suppose that the inequality (3.22) does hold. This implies that for sufficiently small coupling $g$ all non-zero solutions $\lambda$ of equation (3.19) have a negative real part. As the coupling is increased one or more complex conjugate pairs of eigenvalues $\lambda$ may cross the imaginary axis signalling the onset of a Hopf bifurcation in the firing times. It turns out that this provides an important mechanism for the desynchronization of integrate-and-fire neurons with strong synaptic coupling, and leads to the formation of non-phase-locked states characterized by periodic or quasiperiodic variations of the inter-spike intervals (ISIs) on closed orbits. Such states can support a number of different forms of complex dynamics including periodically bursting spike trains and the formation of spatially periodic activity patterns (Bressloff and Coombes 1998b,c). Extensions to the case of oscillatory networks with dendritic interactions have also been studied (Bressloff 1999).

One final observation is that the stability condition (3.22) can also be derived by carrying out a phase reduction of the IF model in the weak coupling regime (Bressloff and Coombes 1999). This involves a nonlinear transformation from the original dynamical variables $V(x, t)$ of equation (2.1) to a set of phase variables $\psi(x, t)$ that are slowly varying functions of time $(\partial \psi / \partial t=\mathcal{O}(g))$. After averaging the evolution equation for $\psi$ over a single natural period $T_{0}$ and performing a rescaling of time, one obtains the phase equation

$$
\frac{\partial \psi(x, t)}{\partial t}=g \int_{-\infty}^{\infty} W\left(x-x^{\prime}\right) K_{T_{0}}\left[\psi\left(x^{\prime}, t\right)-\psi(x, t)\right] d x^{\prime}+\mathcal{O}\left(g^{2}\right) .
$$

Linearizing equation (3.23) about the traveling wave solution $\psi(x, t)=\Omega t+k x$ yields a linear phase equation whose characteristic equation is precisely (3.21). Note that the existence and stability of traveling waves in a generalized version of the phase-equation (3.23), which takes into account space-dependent delays, has been studied elsewhere (Crook et al 1997; Bressloff and Coombes 1997). 


\section{Stability of pulses in the excitable regime}

Consider a single pulse propagating in the excitable regime $I_{0}<1$, with the neuron at position $x$ firing at time $T(x)=x / c_{0}$. We shall first determine the local stability of such a solution directly from equation (2.1), and then indicate how this relates to the stability analysis of section 3 in the long-wavelength limit. Suppose that the firing times are perturbed such that $T(x)=x / c_{0}+u(x)$ with $u(x)=\phi(x)$ for $x \leq 0$ and $\phi(x)$ a prescribed, bounded function on $(-\infty, 0]$. Asymptotic stability then corresponds to the condition $u(x) \rightarrow 0$ as $x \rightarrow \infty$ for arbitrary non-uniform initial data $\phi(x)$. Integrating equation (2.1) over the interval $(-\infty, T(x)]$ gives

$$
\begin{aligned}
\mathrm{e}^{x / c_{0}+u(x)}\left[1-I_{0}\right]= & g \int_{-\infty}^{x / c_{0}+u(x)} \mathrm{e}^{t} \int_{-\infty}^{\infty} W\left(x-x^{\prime}\right) \\
& \times J\left(t-x^{\prime} / c_{0}-u\left(x^{\prime}\right)\right) d x^{\prime} d t .
\end{aligned}
$$

Expanding equation (4.1) to first order in $u(x)$ and imposing equation (2.13) leads to the linear integral equation

$$
\int_{-\infty}^{0} \mathrm{e}^{t} \int_{-\infty}^{\infty} W\left(x-x^{\prime}\right) J^{\prime}\left(t+\left[x-x^{\prime}\right] / c_{0}\right)\left[u(x)-u\left(x^{\prime}\right)\right] d x^{\prime} d t=0
$$

Substitution of a solution of the form $u(x)=\mathrm{e}^{\lambda x}$ into equation (4.2) yields the characteristic equation

$$
\int_{-\infty}^{0} \mathrm{e}^{t} \int_{-\infty}^{\infty} W(x) J^{\prime}\left(t+x / c_{0}\right)\left[\mathrm{e}^{-\lambda x}-1\right] d x d t=0 .
$$

Asymptotic stability then holds if all non-zero solutions $\lambda$ of equation (4.3) have negative real part. Such solutions depend indirectly on the coupling $g$ via the speed $c_{0}$, which satisfies equation (2.13). Equation (4.3) can also be derived by taking a long-wavelength limit of the characteristic equation (3.19) for traveling waves. That is, we set $z=\mathrm{e}^{\lambda T}$, multiply both sides of equation (3.19) by $\mathrm{e}^{-T}$, and take the limit $T \rightarrow \infty$ with $c_{0}=(k T)^{-1}$ fixed. More specifically, equation (3.4) shows that

$$
\lim _{T \rightarrow \infty} B_{m}(x)=\delta_{m,-1} W(x) \int_{-\infty}^{0} \mathrm{e}^{t} J^{\prime}\left(t+x / c_{0}\right) d t .
$$

It then follows from equation (3.11) and (4.4) that

$$
\lim _{T \rightarrow \infty} \mathrm{e}^{-T} \widetilde{B}\left(\mathrm{e}^{\lambda T}, p\right)=\int_{-\infty}^{\infty} \int_{-\infty}^{0} \mathrm{e}^{-i p x-\lambda x / c_{0}} W(x) \mathrm{e}^{t} J^{\prime}\left(t+x / c_{0}\right) d t d x .
$$

Since $\lim _{T \rightarrow \infty} \mathrm{e}^{-T}\left[I_{0}-1+g A\right]=0$, we find that equation (3.19) reduces to equation (4.3) after setting $\lambda \rightarrow \lambda / c_{0}+i p$.

We shall now use the characteristic equation (4.3) to determine the stability of a solitary pulse. Using the fact that $J(t)=0$ for $t \leq 0$, we first rewrite equation (4.3) in the form

$$
\int_{0}^{\infty}\left[\mathrm{e}^{-\lambda x}-1\right] W(x) f\left(x / c_{0}\right) d x=0
$$


where

$$
f(\tau)=\int_{0}^{\tau} \mathrm{e}^{-(\tau-t)} J^{\prime}(t) d t
$$

Let $\lambda=a+i b$ and expand equation (4.6) into real and imaginary parts to obtain the pair of equations

$$
\begin{gathered}
H(a, b) \equiv \int_{0}^{\infty}\left[\mathrm{e}^{-a x} \cos (b x)-1\right] W(x) f\left(x / c_{0}\right) d x=0 \\
G(a, b) \equiv \int_{0}^{\infty} \mathrm{e}^{-a x} \sin (b x) W(x) f\left(x / c_{0}\right) d x=0 .
\end{gathered}
$$

The integrals in equations (4.8) and (4.9) can be evaluated along identical lines to the analysis of equation (2.13). That is, we substitute equations (2.10), (2.3) and (4.7) into (4.8) and (4.9), and then integrate over $x$ and $t$ to obtain a pair of contour integrals. Exploiting the analytical properties of $\widetilde{J}(\omega)$ we find that

$$
\begin{gathered}
H(a, b)=\operatorname{Re} P\left(c_{0}+a+i b\right)-P\left(c_{0}\right) \\
G(a, b)=-\operatorname{Im} P\left(c_{0}+a+i b\right)
\end{gathered}
$$

where

$$
P(z)=\frac{z}{(1+z)} \widetilde{J}(-i z)
$$

Note that $P(z)$ is real when $z$ is real, and $P(z) \rightarrow 0$ as $|z| \rightarrow \infty$.

Suppose that $J(\tau)$ is given by the synaptic delay kernel of equation (2.16). We then have the following theorem concerning the asymptotic stability of solitary waves:

Theorem 2 Let $\mathcal{C}_{+}$and $\mathcal{C}_{-}$denote, respectively, the fast and slow solution branches $c_{0}=c_{ \pm}(g)$, $g \geq g_{s}$, of the self-consistency condition (2.13) for the velocity of a solitary pulse with synaptic delay kernel (2.16), see figure 1. Here $g_{s}$ is the critical coupling for the existence of a solitary wave. We then have the following stability results: (i) The branch $\mathcal{C}_{-}$is unstable for all $\tau_{a}$ and $\alpha$. (ii) The branch $\mathcal{C}_{+}$is stable for all $\alpha$ in the case of zero axonal delays $\left(\tau_{a}=0\right)$. (iii) For non-zero delays and sufficiently fast synapses (large $\alpha$ ), there exists a Hopf bifurcation point $g_{h}$ such that $c_{+}(g)$ is stable (unstable) for $g>g_{h}\left(g_{s} \leq g<g_{h}\right)$.

Proof: We first investigate the stability of the lower branch $\mathcal{C}_{-}$. Differentiation of equations (4.8) and (4.9) with respect to $a$ and $b$ gives

$$
\frac{\partial H(a, 0)}{\partial a}=-\left.\frac{\partial G(a, b)}{\partial b}\right|_{b=0}=-\int_{0}^{\infty} x \mathrm{e}^{-a x} W(x) f\left(x / c_{0}\right) d x
$$




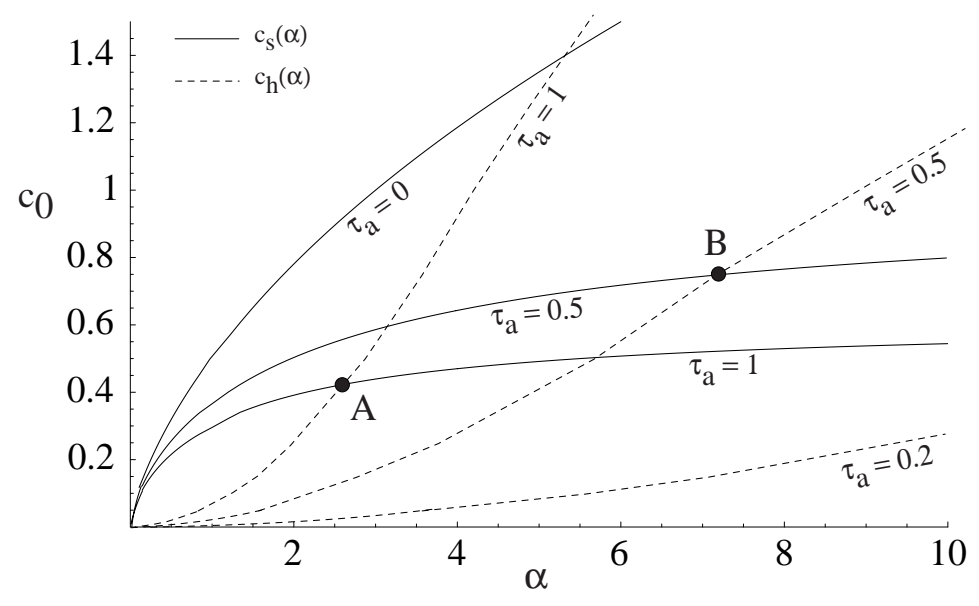

Figure 7: Plot of critical velocities $c_{s}(\alpha)$ and $c_{h}(\alpha)$ as a function of the inverse rise-time $\alpha$ and various axonal delays $\tau_{a}$. (See text for details).

whilst differentiation of equation (2.13) with respect to $g$ yields

$$
\frac{d g}{d c_{0}}=\frac{g^{2}}{c_{0}^{2}} \int_{0}^{\infty} x W(x) f\left(x / c_{0}\right) d x
$$

Comparison of equations (4.13) and (4.14) shows that $\partial H(a, 0) /\left.\partial a\right|_{a=0}>0$ when $d g / d c_{0}<0$, that is, when $c_{0} \in \mathcal{C}_{-}$. Since $\lim _{a \rightarrow \infty} H(a, 0) \equiv-P\left(c_{0}\right)<0$, it follows that $H(a, 0)$ must cross the positive $a$-axis at least once when $c_{0} \in \mathcal{C}_{-}$. This implies that $H(a, 0)=0$ has at least one solution for $a>0$ and, hence, that the branch $\mathcal{C}_{-}$is unstable.

We now determine the stability of the upper branch $\mathcal{C}_{+}$. The delay kernel $J(\tau)$ of equation (2.16) is unimodal with a maximum at $\tau_{\max }=\tau_{a}+\alpha^{-1}$. Hence, the function $f(\tau)$ of equation (4.7) is also unimodal with a maximum at $\tau=\tau_{\max }$ such that $f(\tau)>0$ for $0<\tau<\tau_{\max }$. Since $W(x)$ is a monotonically decreasing function of $x$, it follows from equation (4.8) that $H(a, b)<0$ for all $a>0$ and $b \geq 0$ when $c_{0} \tau_{\max }$ is sufficiently large. In other words, the branch $\mathcal{C}_{+}$is stable in the large- $c_{0}$ limit. It can also be established that $H(a, 0)=0$ does not have any positive definite solutions when $c_{0} \in \mathcal{C}_{+}$so that the upper branch is stable with respect to static bifurcations. Therefore, we investigate possible (Hopf) instabilities of the upper branch by reducing the velocity $c_{0}$ for fixed $\alpha, \tau_{a}$ and searching for solutions $a=0, b \neq 0$ of equations (4.8) and (4.9). Let $c_{h}$ denote the largest velocity for which such a solution exists. Also set $c_{s}=c_{ \pm}\left(g_{s}\right)$, which is the critical velocity at which the two solution branches $\mathcal{C}_{ \pm}$meet. In figure 7 , we plot the critical velocities $c_{s}(\alpha)$ and $c_{h}(\alpha)$ as a function of $\alpha$ and various delays. For a given delay $\tau_{a}, c_{0} \in \mathcal{C}_{+}$if $c_{0}>c_{s}(\alpha)$ and $c_{0} \in \mathcal{C}_{-}$if $c_{0}<c_{s}(\alpha)$. It can be seen from figure 7 that if $\tau_{a} \neq 0$ then there exists a critical inverse rise-time $\alpha_{c}$ such that $c_{s}(\alpha)>c_{h}(\alpha)$ for $\alpha<\alpha_{c}$ and $c_{h}(\alpha)>c_{s}(\alpha)$ for $\alpha>\alpha_{c}$. The crossover points in the particular cases $\tau_{a}=1$ and $\tau_{a}=0.5$ are labelled by $A$ and $B$ respectively. Suppose that we now reduce $c_{0}$ or, equivalently $g$, along the 
branch $\mathcal{C}_{+}$for fixed $\alpha$ and $\tau_{a}>0$. For $\alpha>\alpha_{c}$, we find that a Hopf bifurcation occurs at a critical coupling $g_{h}$ such that $c_{+}\left(g_{h}\right)=c_{h}(\alpha)$ and solitary waves are unstable for all $c_{0}<c_{h}(\alpha)$. On the other hand, if $\alpha<\alpha_{c}$ then the branch $\mathcal{C}_{+}$remains stable until it merges with the unstable lower branch $\mathcal{C}_{-}$at $c_{0}=c_{s}(\alpha)$. This completes the proof.
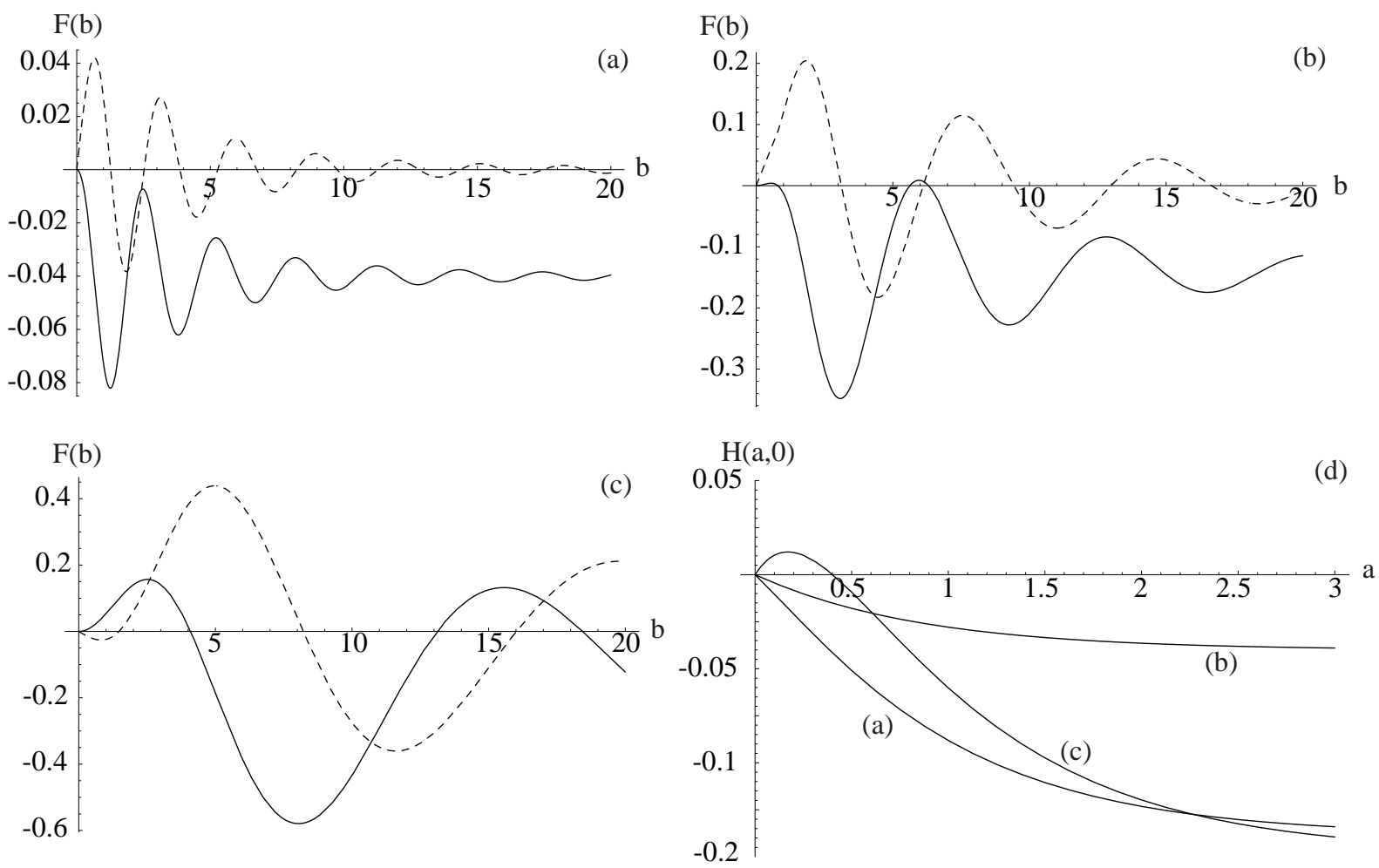

Figure 8: Plot of $F(b)=H(0, b)$ (solid curves) and $F(b)=G(0, b)$ (dashed curves) as a function of $b$ for $\tau_{a}=1, \alpha=4$ and various velocities: (a) $c_{0}=2.0$, (b) $c_{0}=0.8$, (c) $c_{0}=0.3$. The corresponding variation of $H(a, 0)$ with $a$ is shown in figure $8 \mathrm{~d}$. The function $H(a, 0)$ develops a zero on the positive $a$-axis as $c_{0}$ passes through the critical point $c_{s} \approx 0.5$ from the branch $\mathcal{C}_{+}$ to $\mathcal{C}_{-}$. There also exists a Hopf bifurcation point on the branch $\mathcal{C}_{+}$at $c_{h} \approx 0.8$.

Theorem 2 is illustrated in figure 8, where we plot $H(0, b)$ and $G(0, b)$ as a function of $b$ for $\tau_{a}=0, \alpha=2$ and various velocities $c_{0}$. The corresponding variation of $H(a, 0)$ with $a$ is also shown. It can be seen that $H(a, 0)$ has a zero on the positive $a$-axis when $c_{0}<c_{s} \approx 0.4$, and that there exists a Hopf bifurcation point at $c_{h} \approx 0.8$. The fact that axonal delays can induce a Hopf instability for solitary pulses has also been observed by Ermentrout and Golomb (1998). A number of comments are in order.

1. The proof of theorem 2 holds for any monotonically decreasing, positive symmetric weight kernel $W(x)$. For example, rather than the exponential function (2.3), one equally well 
take $W(x)=\mathrm{e}^{-x^{2} / 2 \sigma^{2}} / \sqrt{2 \pi \sigma^{2}}$, or $W(x)=\Theta(\sigma-|x|) / 2 \sigma$.

2. Linear stability analysis is itself insufficient to determine (i) whether the Hopf bifurcation is subcritical or supercritical and (ii) the new state generated by the instability.

3. The results of theorem 2 can be used to conjecture about the stability properties of finite wavelength traveling waves. More specifically, in the case of the dispersion curves shown in figure 2 with $\alpha=2$ and $\tau_{a}=0$, the stability (instability) of the corresponding fast (slow) solitary wave solutions suggests that dispersion curve I is stable, whereas II and III are unstable, at least for sufficiently small $k$. A direct analysis of their stability properties, both in terms of solutions of the full characteristic equation (3.19) and through numerical simulations, is currently under investigation.

4. Theorem 2 can be extended to the case of dendritic interactions with the delay kernel $J(\tau)$ now given by the Green's function $G\left(\xi_{0}, t\right)$ of a semi-infinite dendritic cable, see equation (2.20). Again denote the fast and slow solution branches by $\mathcal{C}_{+}$and $\mathcal{C}_{-}$respectively, see figure 3. Then $\mathcal{C}_{-}$is unstable and $\mathcal{C}_{+}$stable for all $\xi_{0}$; the upper branch cannot destabilize via a Hopf bifurcation unless additional axonal delays are included. It is also possible to take into account active membrane properties under the assumption that variations of the dendritic membrane potential are small so that the channel kinetics can be linearized along the lines developed by Koch (1984). The resulting membrane impedance of the dendrites displays resonant-like behavior, which has recently been shown to induce resonant-like synchronization and bursting effects in networks of oscillatory IF neurons (Bressloff 1999). We have also found that active dendrites can induce Hopf instabilities for solitary pulses in excitable networks.

\section{Two-dimensional networks}

One of the interesting features of two-dimensional excitable media is that it is possible to have waves with non-trivial geometric structures such as spirals and target patterns (Tyson and Keener 1988). A number of recent numerical studies have shown that such waves can also occur in two-dimensional networks of integrate-and-fire neurons (Chu et al 1994; Horn and Opper 1997; Usher et al 1995; Kistler et al 1998). In this final section, we indicate how to extend our analysis of traveling waves in one dimension to the case of plane waves in two-dimensional networks, and relate our results to recent work by Kistler et al (1998) on the so-called spike response model (Gerstner 1995). The case of more complex solutions will be the subject of future work.

A two-dimensional sheet of IF neurons with synaptic coupling evolves according to the equation

$$
\frac{\partial V(\mathbf{r}, t)}{\partial t}=-V(\mathbf{r}, t)+g \int_{\mathbb{R}^{2}} W\left(\left|\mathbf{r}-\mathbf{r}^{\prime}\right|\right) \int_{0}^{\infty} J(\tau) E\left(\mathbf{r}^{\prime}, t-\tau\right) d \tau d^{2} \mathbf{r}^{\prime},
$$


where $\mathbf{r}=(x, y)$ and $E(\mathbf{r}, t)=\sum_{m \in \mathbb{Z}} \delta\left(t-T_{m}(\mathbf{r})\right)$ is the output spike train of the neuron at r. Equation (5.1) is supplemented by the condition that $V(\mathbf{r}, t)$ is reset to zero whenever the neuron at $\mathbf{r}$ reaches threshold $h=1$. For simplicity, we have set the external bias $I_{0}$ to zero (so that the network is operating in the excitable regime). A plane wave solution is defined according to $T_{m}(\mathbf{r})=(m+\mathbf{k} \cdot \mathbf{r}) T$ where $T=T(\mathbf{k})$ is the self-consistent period of oscillations and $\mathbf{k}=\left(k_{1}, k_{2}\right)$ is the wavevector. The speed of the wave is $c=1 /(T|\mathbf{k}|)$. The collective period $T$ is determined from a two-dimensional version of the phase-locking equation (2.5),

$$
1=g \int_{\mathbb{R}^{2}} W(|\mathbf{r}|) K_{T}(\mathbf{k} \cdot \mathbf{r}) d^{2} \mathbf{r} .
$$

Similarly, a solitary pulse propagating with speed $c_{0}$ in the direction $\hat{\mathbf{n}}$ corresponds to a solution in which each neuron fires once with $T(\mathbf{r})=\hat{\mathbf{n}} . \mathbf{r} / c_{0}$. The speed $c_{0}$ satisfies (cf. equation (2.13))

$$
1=g \int_{\mathbb{R}^{2}} \int_{-\infty}^{0} W(|\mathbf{r}|) \mathrm{e}^{t} J\left(t-\hat{\mathbf{n}} \cdot \mathbf{r} / c_{0}\right) d t d^{2} \mathbf{r} .
$$

The stability of plane waves and pulses can be analyzed along identical lines to sections 3 and 4. For the sake of illustration, consider a pulse propagating in the $x$-direction with speed $c_{0}$ so that $\hat{\mathbf{n}}=(1,0)$. By considering perturbations of the firing times, $T(\mathbf{r})=x / c_{0}+u(\mathbf{r})$, we obtain a linear integral equation for $u(\mathbf{r})$ analogous to equation (4.2). This has solutions of the form $u(\mathbf{r})=\mathrm{e}^{\lambda x} \mathrm{e}^{i \mathbf{p} . \mathbf{r}}$ with $\mathbf{p}=\left(p_{1}, p_{2}\right)$ and $\lambda$ satisfying the characteristic equation (see also equation (4.6))

$$
\int_{-\infty}^{\infty} \int_{0}^{\infty} W(|\mathbf{r}|)\left[\mathrm{e}^{-\lambda x} \mathrm{e}^{-i \mathbf{p} . \mathbf{r}}-1\right] f\left(x / c_{0}\right) d x d y=0
$$

In equation (5.4), we can restrict $\lambda$ to be real (due to the presence of the factor $\mathrm{e}^{i \mathbf{p . r}}$ ) so that the condition for asymptotic stability is $\lambda<0$ for all $\mathbf{p} \neq 0$. Similarly, one can derive a twodimensional version of the characteristic equation (3.19) for traveling plane waves by considering perturbations $T_{m}(\mathbf{r})=(m+k x) T+u_{m}(\mathbf{r})$ with $u_{m}(\mathbf{r}) \sim z^{-(n+k x)} \mathrm{e}^{-i \mathbf{p} \cdot \mathbf{r}}$.

It is instructive to compare our stability analysis based on the characteristic equation of the linearized firing time map to the results of Kistler et al (1998). They considered traveling waves and pulses in a network of excitable neurons based on the spike response model (Gerstner 1995). The spike response model is essentially an integrated version of the IF model for which

$$
V(\mathbf{r}, t)=\sum_{m \in \mathbb{Z}} \eta\left(t-T_{m}(\mathbf{r})\right)+g \int_{\mathbb{R}^{2}} \sum_{m \in \mathbb{Z}} W\left(\left|\mathbf{r}-\mathbf{r}^{\prime}\right|\right) \epsilon\left(t-T_{m}\left(\mathbf{r}^{\prime}\right)\right) d^{2} \mathbf{r}^{\prime}
$$

for all $t>0$ with

$$
\eta(t)=-\mathrm{e}^{-t} \Theta(t), \quad \epsilon(t)=\int_{0}^{t} \mathrm{e}^{-\left(t-t^{\prime}\right)} J\left(t^{\prime}\right) d t^{\prime} .
$$

Reset is taken care of by the first term on the right-hand side of equation (5.5). Additional aspects of refractoriness could be incorporated into equation (5.5) by appropriate generalizations 
of $\eta(t)$. These would be particularly important in the case of high frequency traveling waves (small collective period $T$ ), since neurons that had recently fired would still be in their refractory phase when the next wave front arrived. Plane wave solutions are obtained by imposing the appropriate phase-locking ansatz for the firing times in equation (5.5) and setting $V\left(\mathbf{r}, T_{n}(\mathbf{r})\right)=$ 1. The resulting equation for the collective period $T$ is given by (5.2), which can then be used to generate dispersion curves. Note that, in contrast to figures 1 and 2 of the IF model studied in section 2, only a single dispersion curve $\nu=\nu(k)$ was found by Kistler et al (1998) for each set of parameter values, and this corresponded to the continuation of a single long-wavelength solution. The origin of this discrepancy is that Kistler et al (1998) took the activity function $E(\mathbf{r}, t)$ to be of the form $E(\mathbf{r}, t)=\sum_{m \in \mathbb{Z}} \delta(c[t-(x+m) T])$ rather than $E(\mathbf{r}, t)=\sum_{m \in \mathbb{Z}} \delta(t-(k x+m) T)$ as in our own work. This introduces an additional factor of $1 / c$ on the right-hand side of equations (5.2) and (5.5), which leads to a corresponding modification in the dispersion curves. However, the biophysical interpretation of $E(\mathbf{r}, t)$ as an output spike train supports the version adopted by ourselves. It is also the form used by Ermentrout (1998).

In order to determine the stability of a plane wave in the spike response model, Kistler et al (1998) considered the effect of perturbing a single wavefront. That is, they took $T_{n}(\mathbf{r})=$ $(n+k x) T-a \delta_{n, 0} \sin \left(p_{2} y\right)$ for $x<0$ and then used equation (5.4) to calculate the change of potential at $x=0$ to first order in the amplitude $a: \delta V(y)=V\left(0, y, T_{0}(0, y)\right)-V(0,0,0)$. Their condition for local stability was $\delta V\left(\pi / 2 p_{2}\right)<0$, i.e. neurons that have previously fired too early have a reduced potential. For example, in the particular case of a long-wavelength solitary pulse this leads to the condition

$$
\delta V\left(\pi / 2 p_{2}\right)=a g \int_{-\infty}^{\infty} \int_{0}^{\infty} W(|\mathbf{r}|)\left[\cos \left(p_{2} y\right)-1\right] f\left(x / c_{0}\right) d x d y<0,
$$

where we have used the relation $\epsilon^{\prime}(t)=f(t)$, see equations (4.8) and (5.6). Comparison of equation (5.7) with the characteristic equation (5.4) shows that the stability criterion of Kistler et al (1998) does not guarantee asymptotic stability as defined in this paper. Moreover, equation (5.7) does not make sense when the network is restricted to be one-dimensional by setting $W(|\mathbf{r}|)=W(x) \delta(y)$, say. It is also clear that in the case of finite-wavelength plane waves, restricting perturbations of initial data to a single wavefront is a much more restrictive class of Cauchy problem than considered in figure 6 and theorem 1 . In particular, it does not generate the full spectrum of the linearized firing time map as determined by the solutions of the characteristic equation (3.19). As we have pointed out in section 3, details concerning this spectrum are crucial for understanding desynchronization effects in the strong coupling regime.

\section{Acknowledgements}

This work was supported by grant number GR/K86220 from the EPSRC (UK). 


\section{References}

R. D. Chervin, P. A. Pierce and B. W. Connors (1988). Periodicity and directionality in the propagation of epileptiform discharges across neocortex. J. Neurophysiol. 60 1695-1713.

P. C. Bressloff and S. Coombes (1997). Synchrony in an array of integrate-and-fire neurons with dendritic structure. Phys. Rev. Lett. 78, 4665-4668.

P. C. Bressloff and S. Coombes (1998a). Traveling waves in a chain of pulse-coupled oscillators. Phys. Rev. Lett. 80 4815-4818.

P. C. Bressloff and S. Coombes (1998b). Desynchronization, mode-locking and bursting in strongly coupled integrate-and-fire oscillators. Phys. Rev. Lett. 81 2168-2171.

P. C. Bressloff and S. Coombes (1998c). Spike train dynamics underlying pattern formation in integrate-and-fire oscillator networks. Phys. Rev. Lett. 81 2384-2387.

P. C. Bressloff and S. Coombes (1999). Symmetry and phase-locking in a ring of pulse-coupled oscillators with distributed delays. Physica D. 126, 99-122.

P. C. Bressloff (1999). Resonant-like synchronization and bursting in a model of pulse-coupled neurons with active dendrites. J. Comput. Neuro. In press.

P. H. Chu, J. G. Milton and J. D. Cowan (1994). Connectivity and the dynamics of integrateand-fire neural networks. Int. J. Bif. Chaos 4, 237.

S. M. Crook, G. B. Ermentrout, M. C. Vanier, and J. M. Bower. The role of axonal delay in the synchronization of networks of coupled cortical oscillators. J. Comp. Neurosci., 4, 161-172.

A. Destexhe, Z. F. Mainen and T. J. Sejnowski (1994). Synthesis of models for excitable membranes, synaptic transmision and neuromodulation using a common kinetic formalism. J. Comp. Neurosci. 1 195-231.

A. Destexhe, T. Bal, D. A. McCormick and T. J. Sejnowski (1996). Ionic mechanisms underlying synchronized oscillations and propagating waves in a model of ferrety thalamic slices. $J$. Neurophysiol. 76 2049-2070.

G. B. Ermentrout and J. Rinzel (1981). Waves in a simple, excitable or oscillatory, reactiondiffusion model. J. Math. Biol. 11 269-294.

G. B. Ermentrout (1998). The analysis of synaptically generated traveling waves. J. Comp. Neurosci. 5 191-208.

G. B. Ermentrout and D. Golomb (1998). Private communication.

W. Gerstner (1995). Time structure of the activity in neural-network models. Phys. Rev. E 51, 738-758. 
D. Golomb, X. J. Wang and J. Rinzel (1996). Propagation of spindle waves in a thalamic slice model. J. Neurophysiol. 75, 750-769.

D. Golomb and Y. Amitai (1997). Propagating neuronal discharges in neocortical slices: Computational and experimental study. J. Neurophysiol. 78 1199-1211.

D. Horn and I. Opper (1997). Solitary waves of integrate-and-fire neural fields. Neural Comput. 91677.

J. P. Keener, F. C. Hoppensteadt and J. Rinzel (1981). Integrate-and-fire models of nerve membrane response to oscillatory input. SIAM J. Appl. Math. 41 503-517.

U. Kim, T. Bal and D. A. McCormick (1995). Spindle waves are propagating synchronized oscillations in the ferret LGN in vitro. J. Neurophysiol. 74 1301-1323.

W. M. Kistler, R. Seitz and J. L. van Hemmen (1998). Modeling collective excitations in cortical tissue. Physica D 114 273-295.

C. Koch (1984). Cable theory in neurons with active, linearized membranes. Biol. Cybern., 50, $15-33$.

W. Rall (1989). In: C Koch and I Segev (editors), Methods of Neuronal Modeling: From Synapses to Networks, pp.9-62. (MIT Press, Cambridge).

R. D. Traub, J. G. R. Jeffreys and R. Miles (1993). Analysis of propagation of disinhibitioninduced after-discharges along the guinea-pig hippocampal slice in vitro. J. Physiol. (London) 472 267-287.

J. J. Tyson and J. P. Keener (1988). Singular perturbation theory of traveling waves. (A review). Physica D 32, 327-361.

M. Usher, M. Stemmler and Z. Olami (1995). Dynamic pattern formation leads to $1 / f$ noise in neural populations. Phys. Rev. Lett. 74, 326. 